\title{
Chaos and band structure in a three-dimensional optical lattice
}

\author{
Yingyue Boretz and L. E. Reichl \\ The Center for Complex Quantum Systems, The University of Texas at Austin, Austin, Texas 78712
}

(Received 22 December 2014; published 3 April 2015)

\begin{abstract}
Classical chaos is known to affect wave propagation because it signifies the presence of broken symmetries. The effect of chaos has been observed experimentally for matter waves, electromagnetic waves, and acoustic waves. When these three types of waves propagate through a spatially periodic medium, the allowed propagation energies form bands. For energies in the band gaps, no wave propagation is possible. We show that optical lattices provide a well-defined system that allows a study of the effect of chaos on band structure. We have determined the band structure of a body-centered-cubic optical lattice for all theoretically possible couplings, and we find that the band structure for those lattices realizable in the laboratory differs significantly from that expected for the bands in an "empty" body-centered-cubic crystal. However, as coupling is increased, the lattice becomes increasingly chaotic and it becomes possible to produce band structure that has behavior qualitatively similar to the "empty" body-centered-cubic band structure, although with fewer degeneracies.
\end{abstract}

DOI: 10.1103/PhysRevE.91.042901

PACS number(s): 05.45.Ac, 42.50.Wk, 05.45.Gg

\section{INTRODUCTION}

The effect of classical chaos on wave motion can be seen most directly when considering the behavior of eigenvalues and eigenfunctions of the Schrodinger equation for a particle in a potential well [1]. When the particle has two degrees of freedom, one can directly compare Poincare surfaces of section (PSS) of the classical motion to quantum surfaces of section of Husimi functions built from eigenstates of the Schrodinger equation. The effect of chaos on quantum systems with more than two degrees of freedom is more difficult to determine in a systematic way because it is not easy to visualize either classical or quantum motion in systems with more than two degrees of freedom. The effects of chaos can also be seen in other types of wave propagation. Electromagnetic radiation exhibits the manifestations of chaos and this has been measured experimentally in microwaves [2,3]. Acoustic waves exhibit the effects of chaos and this has special importance in the study of sound wave motion in the ocean [4].

When waves propagate through a potential energy field that forms a periodic array, the allowed energies or frequencies form bands of values for which waves are allowed to propagate and gaps between the allowed energy bands for which no wave propagation can occur. In crystalline solids, formed from periodic arrays of atoms or molecules, electron matter waves can propagate and the detailed structure of electron energy bands and band gaps determines whether the material is a conductor, semiconductor, or an insulator of electricity [5]. Photonic crystals can be fabricated and consist of regularly repeating regions of low and high dielectric constant. Photonic crystals allow the propagation of electromagnetic waves whose wavelength is of the order of periodicity of the dielectric lattice. Electromagnetic waves have a scale invariance so that electromagnetic waves of any wavelength can propagate in a photonic crystal, provided the periodicity of the crystal is the same order as the wavelength of the radiation. Electromagnetic waves in photonic crystals have bands of allowed wavelengths that can propagate and band gaps in which no radiation can propagate $[6,7]$. Elastic and acoustic waves through materials with spatially periodic arrays of material, with different elastic moduli, have bands of allowed wavelengths in which the acoustic waves can propagate and band gaps in which waves are not allowed to propagate [8].

One of the most recent additions to this collection of systems with wave propagation in periodic lattices is the propagation of atomic matter waves (alkali atoms such as rubidium, cesium, and sodium) in optical lattices. Optical lattices are formed by superposing multiple pairs of counterpropagating laser beams in different patterns and have been realized in the laboratory by several groups [9-13]. Such systems can be used to explore the quantum-classical correspondence of the atoms interacting with the optical lattice, as was done in Ref. [14] for a $2 \mathrm{D}$ optical lattice and, by analogy, some properties of electrons in crystalline solids $[15,16]$.

In subsequent sections, we use a dynamical model that, for a particular parameter range, describes the interaction of alkali atoms with a three-dimensional (3D) optical lattice. However, the model more generally allows us to explore how the detailed structure of the periodic lattice can affect band structure. As was shown in Ref. [14], the coupling between degrees of freedom of the lattice can be varied by varying the relative orientation of the polarization of the lasers. As a consequence, the nature of the particle dynamics can be varied from integrable behavior to almost fully chaotic behavior for a range of energies. This transition from integrable to chaotic behavior occurs because resonances between the degrees of freedom in the coupled system destroy local constants of the motion [1], thus allowing the particles to access larger regions of the lattice. In bounded systems, this transition is known to have a profound effect of the dynamics of quantized system. Below we show how the transition to chaos can affect the energy band structure of particles confined to the 3D BCC optical lattice.

We begin in Sec. II with a description of the Hamiltonian and dynamics of atoms confined to a 3D optical lattice. In Sec. III, we describe the classical dynamics of noninteracting particles on the lattice and discuss properties of invariant manifolds embedded in the 3D lattice. In Sec. IV, we describe the quantum dynamics of particles on the lattice and show the effect of chaos on the energy band structure. Finally, in Sec. V, we make some concluding remarks. 


\section{THREE-DIMENSIONAL OPTICAL LATTICE}

We consider a gas of noninteracting particles of mass $m$ interacting with a body-centered-cubic (BCC) lattice formed by the superposition of three pairs of mutually perpendicular laser beams (see Appendix). The laser beams are detuned from resonance with the atoms, so the interaction dynamics between the atoms and the optical lattice can be described by the Hamiltonian [17]

$$
\begin{aligned}
H_{\mathrm{eff}}= & \frac{p_{z}^{2}}{2 m}+\frac{p_{y}^{2}}{2 m}+\frac{p_{z}^{2}}{2 m}-\frac{d_{A}^{2} \mathcal{E}^{2}}{\hbar \Delta}\left[\cos ^{2}\left(k_{x} x\right)+\cos ^{2}\left(k_{y} y\right)\right. \\
& +\cos ^{2}\left(k_{z} z\right)+2 a \cos \left(k_{x} x\right) \cos \left(k_{y} y\right) \\
& \left.+2 b \cos \left(k_{x} x\right) \cos \left(k_{z} z\right)+2 c \cos \left(k_{y} y\right) \cos \left(k_{z} z\right)\right],
\end{aligned}
$$

where $\hbar$ is Planck's constant, $x, y$, and $z$ are the particle Cartesian coordinates, $p_{x}, p_{y}$, and $p_{z}$ are components of the particle momentum in the $x, y$, and $z$ directions, respectively, $d_{A}$ is the atomic dipole moment, $\mathcal{E}$ is the laser electric field strength, $k_{x}, k_{y}$, and $k_{z}$ are the wave vectors of the laser beams in the $x, y$, and $z$ directions, respectively, and $\Delta$ is the detuning of the laser from resonance with the atoms. The coupling constants, $a, b$, and $c$ (with the lasers in phase), are given by $a \equiv \hat{\epsilon}^{(x)} \cdot \hat{\epsilon}^{(y)}, b \equiv \hat{\epsilon}^{(x)} \cdot \hat{\epsilon}^{(z)}$, and $c \equiv \hat{\epsilon}^{(y)} \cdot \hat{\epsilon}^{(z)}$, where $\hat{\epsilon}^{(x)}, \hat{\epsilon}^{(y)}$, and $\hat{\epsilon}^{(z)}$ are the polarization vectors for lasers directed along the $x, y$, and $z$ axes, respectively. Thus, the coupling constants are determined by the physically allowed angles between the polarization directions of the laser fields.

In Fig. 1, we show a plot of the polarization vectors and the angles these vectors make with respect to the Cartesian coordinate frame. The vector $\hat{\epsilon}^{(x)}$ lies in the $y-z$ plane and makes angle $\theta_{x}$ with the $z$ axis. Similarly, $\hat{\epsilon}^{(y)}\left(\hat{\epsilon}^{(z)}\right)$ lies in the $x-z(x-y)$ plane and makes angle $\theta_{y}\left(\phi_{z}\right)$ with the $z$ axis $\left(x\right.$ axis). Since $\hat{\epsilon}^{(x)}=\cos \left(\theta_{x}\right) \hat{z}+\sin \left(\theta_{x}\right) \hat{y}, \hat{\epsilon}^{(y)}=\cos \left(\theta_{y}\right) \hat{z}+$ $\sin \left(\theta_{y}\right) \hat{x}$, and $\hat{\epsilon}^{(z)}=\cos \left(\phi_{z}\right) \hat{x}+\sin \left(\phi_{z}\right) \hat{y}$, we can write $a=$ $\hat{\epsilon}^{(x)} \cdot \hat{\epsilon}^{(y)}=\cos \left(\theta_{x}\right) \cos \left(\theta_{y}\right), b=\hat{\epsilon}^{(x)} \cdot \hat{\epsilon}^{(z)}=\sin \left(\theta_{x}\right) \sin \left(\phi_{z}\right)$, and $c=\hat{\epsilon}^{(y)} \cdot \hat{\epsilon}^{(z)}=\sin \left(\theta_{y}\right) \cos \left(\phi_{z}\right)$.

In subsequent sections, we will consider the case $a=$ $b=c$. Zero coupling between the lasers $a=b=c=0$ corresponds to angles $\theta_{x}=\frac{\pi}{2}, \theta_{y}=0$, and $\phi_{z}=0$. In this case, the polarization vectors form $90^{\circ}$ angles with respect to one another. The maximum physically allowed coupling

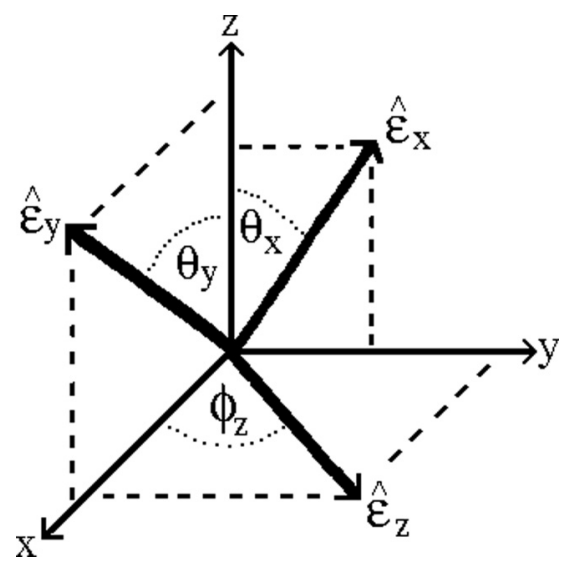

FIG. 1. The directions of the polarization angles relative to the Cartesian axes containing the laser beams. between the laser beams occurs for $a=b=c=\frac{1}{2}$ with angles $\theta_{x}=\theta_{y}=\phi_{z}=\frac{\pi}{4}$. However, from the point of view of dynamics, the cases $\frac{1}{2}<a \leqslant 1$ are also of interest (even though they cannot be realized in the laboratory by the optical lattice), because they tell us the type of dynamics the optical lattice cannot achieve.

We will consider the case where all lasers have the same wavelength so that $k_{x}=k_{y}=k_{z}=k_{L}$. The Hamiltonian then takes the form

$$
\begin{aligned}
H_{\mathrm{eff}}= & \frac{p_{x}^{2}}{2 m}+\frac{p_{y}^{2}}{2 m}+\frac{p_{z}^{2}}{2 m}-\frac{d_{A}^{2} \mathcal{E}^{2}}{\hbar \Delta}\left[\cos ^{2}\left(k_{L} x\right)+\cos ^{2}\left(k_{L} y\right)\right. \\
& +\cos ^{2}\left(k_{L} z\right)+2 a \cos \left(k_{L} x\right) \cos \left(k_{L} y\right) \\
& \left.+2 a \cos \left(k_{L} x\right) \cos \left(k_{L} z\right)+2 a \cos \left(k_{L} y\right) \cos \left(k_{L} z\right)\right] .
\end{aligned}
$$

Let us now introduce dimensionless variables. We measure energy in terms of the recoil energy $E_{L}=\frac{\hbar^{2} k_{L}^{2}}{2 m}$, and let $H_{\mathrm{eff}}=H^{\prime} E_{L}, p_{x}=p_{x}^{\prime}\left(\hbar k_{L}\right), p_{y}=p_{y}^{\prime}\left(\hbar k_{L}\right), p_{z}=p_{z}^{\prime}\left(\hbar k_{L}\right)$, $x^{\prime}=k_{L} x, y^{\prime}=k_{L} y, z^{\prime}=k_{L} z$, and $u E_{L}=-\frac{d_{A}^{2} \mathcal{E}^{2}}{\hbar \Delta}$. Then the Hamiltonian takes the form

$$
H=p_{x}^{2}+p_{y}^{2}+p_{z}^{2}+V(x, y, z)=E,
$$

with

$$
\begin{aligned}
V(x, y, z)= & u\left[\cos ^{2}(x)+\cos ^{2}(y)+\cos ^{2}(z)\right] \\
& +2 a u[\cos (x) \cos (y)+\cos (x) \cos (z) \\
& +\cos (y) \cos (z)],
\end{aligned}
$$

where all quantities are in dimensionless units (d.u.) (we have dropped the primes). We will consider the dynamics for the case $u=20$ d.u., which is the case considered in Ref. [14] and is a value attainable in experiment.

In subsequent sections, we will focus on three representative values of the coupling constant, $a=0.1, a=0.5$, and $a=1.0$. For all three values of the coupling constant, the optical lattice forms a body-centered cubic (BCC) structure and the minimum value of the potential energy is $V(x, y, z)=0$ for $x, y$, and $z$ each taking all possible values $(2 m+1) \pi / 2$ (integer $m$ can have values $-\infty \leqslant m \leqslant \infty$ ). For all three cases, the maximum value of the potential energy $V(x, y, z)=V_{m x}(a)$ is located at values of $x, y$, and $z$ each taking values $m \pi$, where integer $m$ can have values $-\infty \leqslant m \leqslant \infty$. However, the values of $V_{m x}(a)$ are different: $V_{m x}(0.1)=72$ d.u., $V_{m x}(0.5)=$ 120 d.u., and $V_{m x}(1.0)=180$ d.u. Also, the detail structure of the potential energy surfaces is very different in the three cases. In Fig. 2, we show the structure of the potential energy surface for both low energy and high energy (up to the maximum value of the potential energy). At low energy, the lattices for $a=0.1$ and $a=0.5$ have very similar structure, and trajectories are confined within the unit cells (the low-energy values are determined by invariant manifolds that separate unit cells as discussed below). This low-energy structure is particularly significant because, at low temperature, this is the energy region occupied by ultracold gases. At low energy, the lattice for $a=1.0$ is open and trajectories have the ability to move between unit cells. For high energy, the lattices for $a=0.5$ and $a=1.0$ have very similar open structure, while the lattice for $a=0.1$ is much more cluttered with potential energy surfaces. 


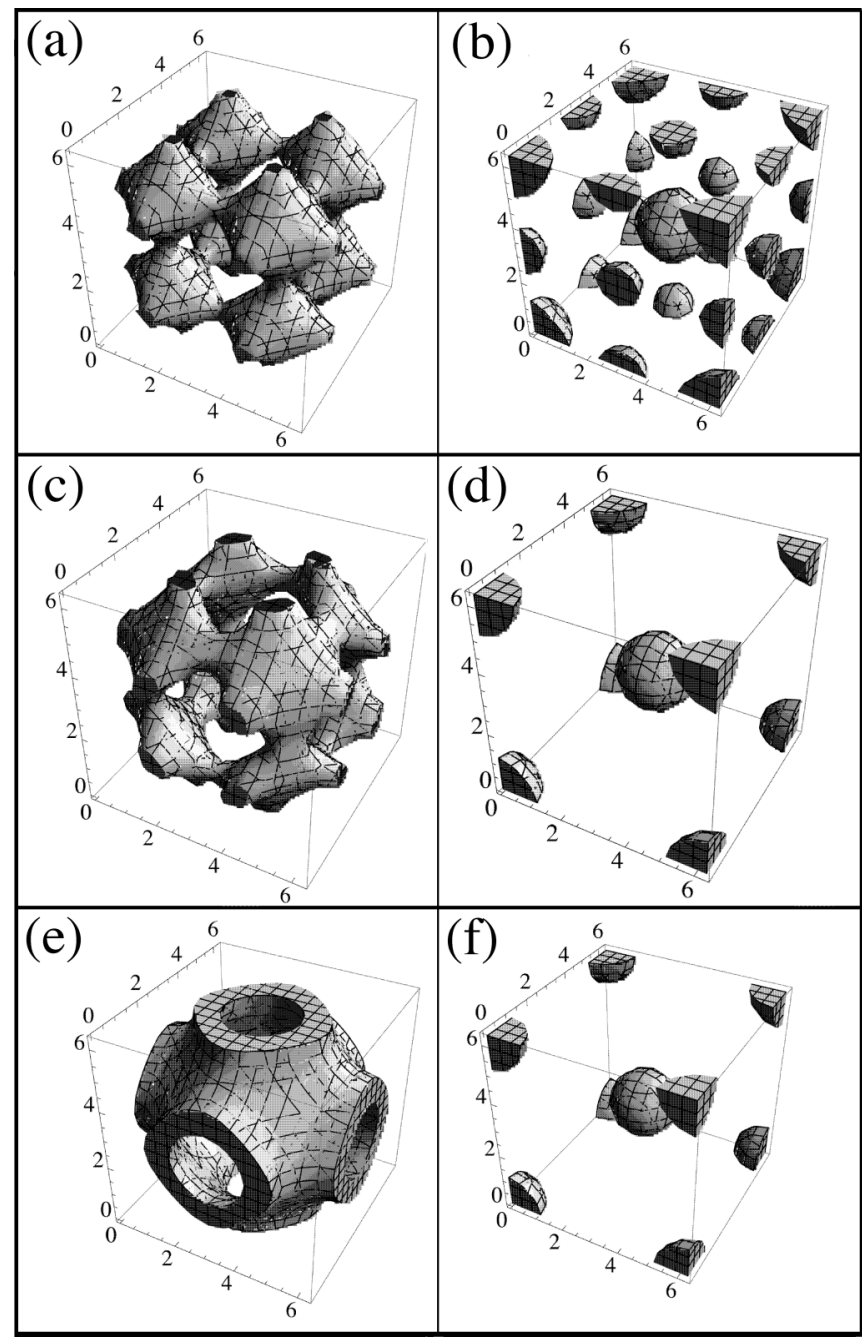

FIG. 2. The 3D potential energy surface for one unit cell of the 3D lattice for different energy intervals: (a) $a=0.1$ and $V(x, y, z)<21.64$ d.u.; (b) $a=0.1$ and $48<V(x, y, z)<72$ d.u.; (c) $a=0.5$ and $V(x, y, z)<15.33$ d.u.; (d) $a=0.5$ and $80<$ $V(x, y, z)<120$ d.u.; (e) $a=1.0$ and $V(x, y, z)<3.0$ d.u.; (f) $a=$ 1.0 and $120<V(x, y, z)<180$ d.u..

In subsequent sections, we consider the classical and quantum dynamics of noninteracting particles confined to this 3D optical lattice.

\section{CLASSICAL DYNAMICS ON THE THREE-DIMENSIONAL LATTICE}

The first step in describing the classical dynamics of particles on this optical lattice is to write Hamilton's equations of motion for the particles. They take the form

$$
\begin{aligned}
\dot{p}_{x} & =[2 u \cos (x)+2 a u \cos (y)+2 a u \cos (z)] \sin (x), \\
\dot{p}_{y} & =[2 u \cos (y)+2 a u \cos (x)+2 a u \cos (z)] \sin (y), \\
\dot{p}_{z} & =[2 u \cos (z)+2 a u \cos (x)+2 a u \cos (y)] \sin (z), \\
\dot{x} & =2 p_{x}, \quad \dot{y}=2 p_{y}, \quad \dot{z}=2 p_{z} .
\end{aligned}
$$

From these equations, we see that the phase space contains three sets invariant manifolds. If we choose initial conditions $\left(p_{x}=0, x=2 m \pi\right)$ (for integers $-\infty \leqslant m \leqslant \infty$ ), then subsequent trajectories will always remain on the twodimensional (2D) invariant manifold (InM) consisting of variables $\left\{p_{y}, p_{z}, y, z\right\}$. Similarly, if we choose $\left(p_{y}=0, y=\right.$ $2 m \pi)\left[\left(p_{z}=0, z=2 m \pi\right)\right]$ the subsequent motion will remain in the InM $\left\{p_{x}, p_{z}, x, z\right\}\left(\left\{p_{x}, p_{y}, x, y\right\}\right)$. The dynamics on these three InMs will be identical functions of the coupling constant $a$ (for the case $a=b=c$ considered here). The InMs play a critical role in the dynamics of the 3D lattice. They form the boundaries between different unit cells and determine the flow of trajectories between different unit cells of the 3D lattice.

Because the dynamics on the InMs evolves in a $2 \mathrm{D}$ phase space, it is possible to visualize the dynamics on the InMs using Poincare surfaces of section (PSS). This provides valuable information about the character of the dynamics on the lattice that is not readily accessible in the $3 \mathrm{D}$ lattice. In the sections below, we first analyze the dynamics on the InMs and then consider the full 3D dynamics of the system.

\section{A. Invariant manifolds}

The dynamics on the three sets of InMs are identical functions of the coupling constant $a$, so we will focus on the InM generated by setting $p_{x}=0, x=0$. The Hamiltonian that describes the dynamics of the InM is

$$
H_{\mathrm{IM}}=p_{y}^{2}+p_{z}^{2}+V_{\mathrm{IM}}(y, z)=E_{\mathrm{IM}},
$$

where

$$
\begin{aligned}
V_{\mathrm{IM}}(y, z)= & u+u\left[\cos ^{2}(y)+\cos ^{2}(z)\right] \\
& +2 a u[\cos (y)+\cos (z)+\cos (y) \cos (z)] .
\end{aligned}
$$

In Figs. 3(a), 3(b), and 3(c), we show plots of one unit cell of the potential energy surface on the InM for $a=0.1,0.5,1.0$, respectively.

The potential energy surface on the InM undergoes a significant change in its structure as we increase the coupling constant. For $a<1.0$, the InM always has its potential energy maxima $V_{\max }(a)$ at positions $y=2 \pi m, z=2 \pi n$, where $(m, n=0, \pm 1, \ldots)$, and it has potential energy local maxima $V_{\operatorname{lmx}}(a)<V_{\max }(a)$ at points $y=c_{y} \pi+2 \pi m, z=c_{z} \pi+2 \pi n$, where $\left(c_{y}, c_{z}\right)=(1,1),(1,0)$, and $(0,1)$. There are two types of saddle point on the InM. There is a high saddle, with potential energy $V_{\mathrm{hsd}}(a)$, at $y=\frac{\pi}{2} d_{y}+2 \pi m, z=\frac{\pi}{2} d_{z}+2 \pi n$, where $\left(d_{y}, d_{z}\right)=(0,1)$ and $(1,0)$. There is a low saddle, with potential energy $V_{\mathrm{lsd}}(a)<V_{\mathrm{hsd}}(a)$, at $y=\frac{\pi}{2} f_{y}+m \pi, z=\frac{\pi}{2} f_{z}+n \pi$, where $\left(f_{y}, f_{z}\right)=(2,1)$ and $(1,2)$. The minimum value of the potential energy $V_{\min }(a)$ occurs at $y=z=\cos ^{-1}\left[\frac{-a}{(1+a)}\right]$.

The structure of the InM is different for $a=1$. The potential energy maximum and local maximum are $V_{\max }=$ 180 d.u. and $V_{\operatorname{lmx}}=20$ d.u., respectively. There is a high saddle $V_{\text {hsd }}=80$ d.u., but the low saddle merges with the potential energy minimum so that $V_{\text {led }}=V_{\min }=0$. Values of $V_{\max }(a), V_{\operatorname{lmx}}(a), V_{\min }(a), V_{\text {had }}(a), V_{\mathrm{lsd}}(a)$ are given in Table I for $a=0.1,0.5,1.0$.

Trajectories on the InM are confined to the InM unit cell as long as their energy lies below the energy of the high saddle point, or the local potential maximum, whichever has lowest energy. For $a=0.1$, trajectories can escape from the InM unit cell, and traverse the entire InM, by passing over the high saddle point when their energy is greater than $E=44$ d.u.. 


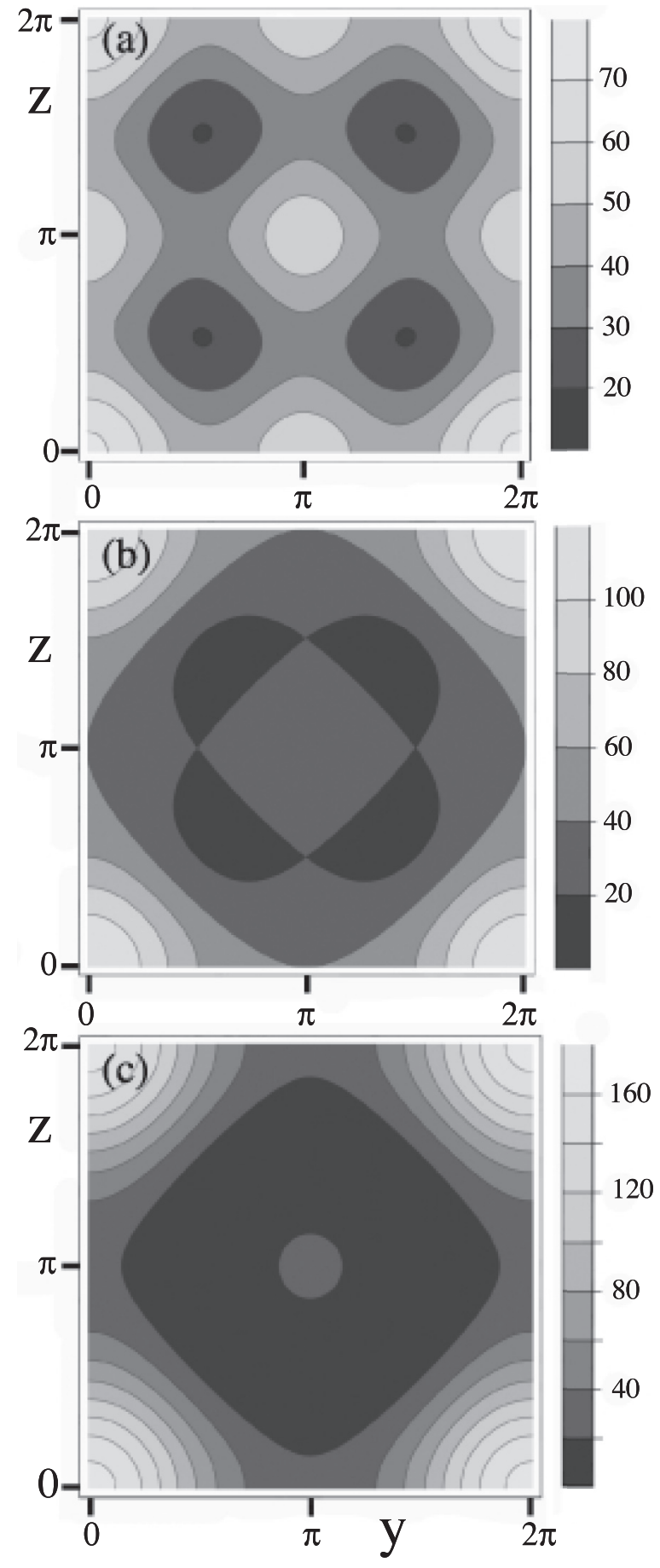

FIG. 3. The potential energy surface $V_{\mathrm{IM}}(y, z)$ for one unit cell the invariant manifold ( $x=0, p_{x}=0$ ) for coupling constants (a) $a=0.1$, (b) $a=0.5$, and (c) $a=1.0$. The energy scale of the shadings is shown in dimensionless units.

TABLE I. Key potential energy values on the InM (in d.u.).

\begin{tabular}{ccccccc}
\hline \hline & $V_{\text {max }}$ & $V_{\text {lmax }}$ & $V_{\text {hisd }}$ & $V_{\text {lwsd }}$ & $V_{\min }$ & $\cos ^{-1}\left(\frac{-a}{1+a}\right)$ \\
\hline$\alpha=0.1$ & 72 & 56 & 44 & 36 & 19.6364 & 1.6618 \\
$\alpha=0.5$ & 120 & 40 & 60 & 20 & 13.3333 & 1.9106 \\
$\alpha=1.0$ & 180 & 20 & 80 & 0 & 0 & 2.0944 \\
\hline \hline
\end{tabular}

For $a=0.5$, trajectories can escape the InM unit cell by passing over the local potential maximum when their energy is greater than $E=40$ d.u.. For $a=1.0$, trajectories can escape the unit cell and move throughout the InM by passing over the local potential maximum when their energy is greater than $E=20$ d.u..

We can visualize the nature of the dynamics on the InM using Poincare surfaces of section (PSS). In Fig. 4 we show a PSS of $p_{z}$ versus $z$, plotted every time $y=y_{f}=\cos ^{-1}\left(\frac{-a}{1+a}\right)$. We use periodic boundary conditions so a trajectory that is able to leave the unit cell enters on the opposite side. Figures 4(a)-4(c) show surfaces of section for $a=0.1$ and a range of energies. In Fig. 4(a), the energy is $E=31$ d.u., which is just below $V_{\text {lsd }}(0.1)=36$ d.u. We see the same bifurcation that occurred in Ref. [14]. With increasing energy, this bifurcation gives rise to a growing chaotic sea in its neighborhood. In Fig. 4(b), the energy is $E=41$ d.u., which is below the high saddle $V_{\text {hsd }}(0.1)=44$ d.u. but above the low saddle $V_{\mathrm{lsd}}(0.1)=36 \mathrm{~d}$.u. The trajectory is able to cross the low saddle but is still confined inside the unit cell. In this figure the chaos associated with the saddle is evident. Indeed, the saddle chaos has mixed with chaotic orbits due to the bifurcation, in a manner similar to that seen in Ref. [14]. In Fig. 4(c), the energy is $E=51$ d.u., which is just above the high saddle $V_{\text {hsd }}(0.1)=44$ d.u., but still below the potential energy maximum $V_{\max }(0.1)=72 \mathrm{~d}$.u.. The trajectory can now escape the unit cell.

In Figs. 4(d)-4(f), we show PSSs for $a=0.5$, which is the maximum value of $a$ that can be achieved in the laboratory for the optical lattice we consider here. In Fig. 4(d), the energy is $E=18$ d.u., which is just below the low saddle $V_{\text {lsd }}(0.5)=20$ d.u.. The structure is quite different from that seen in Ref. [14], because the InM is a cubic lattice and the 2D lattice in Ref. [14] is a body-centered cubic. This figure shows a bifurcation in the upper KAM island. In Fig. 4(e), the energy is $E=24$ d.u., which is below the high saddle $V_{\text {hsd }}(0.5)=60$ d.u. but above the low saddle $V_{\text {lsd }}(0.5)=$ 20 d.u.. The trajectory is able to cross the low saddle but is still confined inside the unit cell. Finally, in Fig. 4(f), the energy is $E=64$ d.u., which is just above the high saddle $V_{\text {hsd }}(0.5)=64$ d.u., but still well below the potential energy maximum $V_{\max }(0.5)=120 \mathrm{~d}$.u. The phase space appears to be fully chaotic and trajectories can leave the unit cell and travel through out the InM.

In Figs. 4(g)-4(i), we show PSSs for $a=1.0$. In Fig. 4(g), the energy is $E=1 \mathrm{~d}$.u., which is just above the minimum potential energy $V_{\min }(1.0)=0$. At this very low energy there is still a stable periodic orbit corresponding to flow in the potential energy minimum $V_{\min }=0$ that forms a circular trough in the InM. In Fig. 4(h), the energy is $E=44$ d.u., which is above the local potential energy maximum $V_{\operatorname{lmx}}(1.0)=20$ d.u., but below the high saddle point energy $V_{\text {had }}(1.0)=80 \mathrm{~d}$.u. The motion appears to be fully chaotic but is still confined to the unit cell. Finally, in Fig. 4(i), the energy is $E=84$ d.u., which is just above the high saddle energy $V_{\text {had }}(1.0)=80$ d.u. The trajectories can now leave the unit cell and appear to be fully chaotic, except for a KAM island structure in the neighborhood of the high saddle. As discussed in [14], dynamics on the lattice for $a=1.0$ for $0<E<180$ d.u. has features similar to a K-flow. 

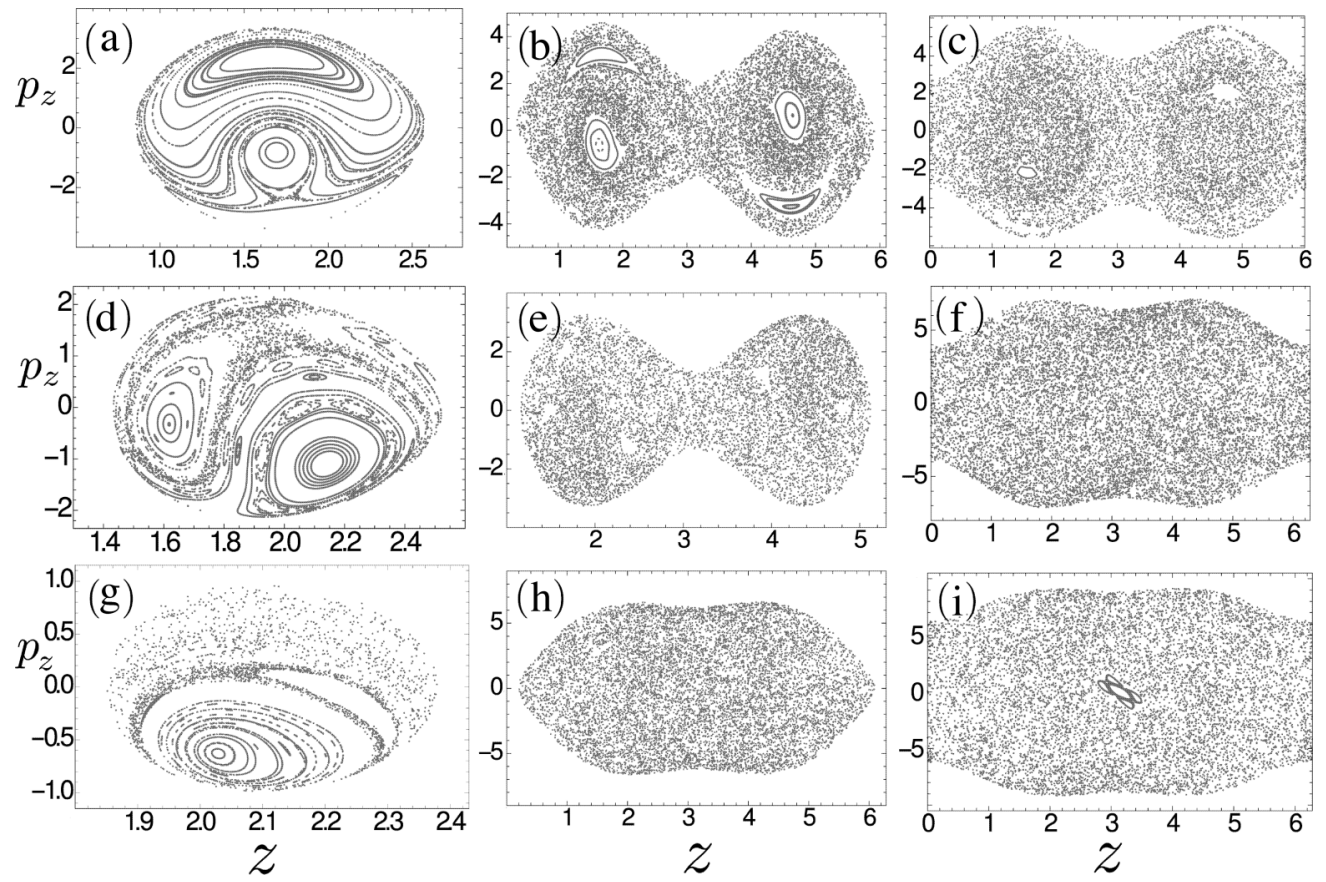

FIG. 4. Poincare surfaces of section of $p_{z}$ versus $z$ for intersections condition $y=\cos ^{-1}\left(\frac{-a}{1+a}\right)$ on the invariant manifold $p_{x}=0, x=0$ : (a) $a=0.1, E=31$ d.u.; (b) $a=0.1, E=41$ d.u.; (c) $a=0.1, E=51$ d.u.; (d) $a=0.5, E=18$ d.u.; (e) $a=0.5, E=24$ d.u.; (f) $a=0.5$, $E=64$ d.u.; (g) $a=1.0, E=1.0$ d.u.; (h) $a=1.0, E=44$ d.u.; (i) $a=1.0, E=84$ d.u..

We can determine the stability of these InMs in directions normal to the InM. Let us consider the InM obtained by setting $\left(p_{x}=0, x=0\right)$. We can expand the 3D Hamiltonian about $x=0$ and obtain

$$
H_{\perp}=H_{\mathrm{IM}}\left(p_{y}, p_{z}, y, z\right)+p_{x}^{2}+\frac{1}{2} k(x, y) x^{2}+\ldots,
$$

where the "force constant" $k(y, z)$ is

$$
k(y, z)=-u[1+a \cos (y)+a \cos (z)] .
$$

The force constant $k(y, z)$ is negative for coupling constant $0 \leqslant a \leqslant 0.5$, and therefore for $0 \leqslant a \leqslant 0.5$, the dynamics normal to the InM is unstable and the InM forms a saddle in the normal direction. If the normal stability is greater than the tangential instability on the InM, then the InM is called a normally hyperbolic invariant manifold (NHIM) [18]. For values of the coupling constant $a \geqslant 0.5$ the stability begins to change in the neighborhood of $y=\pi, z=\pi$ and the force constant becomes positive in a growing region of the InM, in the neighborhood of $y=\pi, z=\pi$, as $a$ further increases.

\section{B. 3D lattice}

The six InMs, located at $x=0,2 \pi, y=0,2 \pi$, and $z=$ $0,2 \pi$, form the outer walls of the unit cell of the 3D lattice. At low energy, they create barriers that trajectories must cross to escape the unit cell. For all values of the coupling constant, the minimum value of potential energy inside the 3D unit cell is $V_{\min }^{3 \mathrm{D}}(a)=0$. For $a \leqslant 0.5$, the InMs are hyperbolic in the normal direction. The height of the saddle is equal to $V_{\min }(a)$ $\left[V_{\min }(0.1)=19.64\right.$ d.u., $V_{\min }(0.5)=13.33$ d.u., $V_{\min }(1.0)=$ $0]$, the minimum value of the potential energy on the InM. For $E<V_{\min }(a)$ trajectories are trapped inside the 3D unit cell. In Figs. 2(a), 2(c), and 2(e), we show the regions of potential energy inside the 3D unit cell with potential energy $V^{3 \mathrm{D}}(a)<\left[V_{\min }(a)+2\right]$ d.u. For $a=0.1$ and $a=0.5$ there is a very small passageway between the 3D unit cells. For $a=1.0$, there is large pipe-shaped region of the phase space connecting them.

The dynamics on the InM gives us an indication of the nature of the dynamics in the 3D unit cell, and is easy to visualize using a PSS. In the 3D unit cell, the available phase space is six-dimensional and the dynamics lives on a five-dimensional energy surface. It is possible to construct a PSS, but it is four-dimensional and cannot be visualized easily. However, we can compute the stability of phase space flow on both the InM and the 3D lattice by finding the Lyapunov exponents associated with the flow. Lyapunov exponents give a measure of the rate of divergence (indicating chaos) or convergence of neighboring trajectories in phase space.

For bounded systems or unbounded systems $[1,19,20]$, the dominant Lyapunov exponent can be obtained by computing the average of a sequence of distances, $d_{j}(j=0,1, \ldots N)$ between neighboring phase space trajectories, each computed for a finite length of time. One starts with two neighboring points in phase space, whose displacements at $t=0$ are given by $\mathbf{X}_{0,0}$ and $\mathbf{Y}_{0,0}$. Initially, $\mathbf{Y}_{0,0}=\mathbf{X}_{0,0}+\mathbf{d}_{0}$, where $\mathbf{d}_{0}$ is the initial displacement between the two points. The two initial points evolve according to Hamilton's equations. After time interval $\tau$, the initial points $\mathbf{X}_{0,0}$ and $\mathbf{Y}_{0,0}$ evolve to $\mathbf{X}_{0, \tau}$, and $\mathbf{Y}_{0, \tau}$, respectively, where $\mathbf{Y}_{0, \tau}=\mathbf{X}_{0, \tau}+\mathbf{d}_{1}$, and the distance between the two points is $d_{1}=\left|\mathbf{d}_{1}\right|$. After this first time step $\tau$, we reset the position of our reference trajectory so that $\mathbf{Y}_{1,0}=\mathbf{X}_{1,0}+\frac{d_{0}}{d_{1}} \mathbf{d}_{1}$, where $\mathbf{X}_{1,0}=\mathbf{X}_{0, \tau}$. We then evolve the two points $\mathbf{X}_{1,0}$ and $\mathbf{Y}_{1,0}$ for one time step $\tau$ and obtain second set of points $\mathbf{X}_{1, \tau}$ and $\mathbf{Y}_{1, \tau}$, where $\mathbf{Y}_{1, \tau}=\mathbf{X}_{1, \tau}+\mathbf{d}_{2}$. We again reset the reference trajectory 


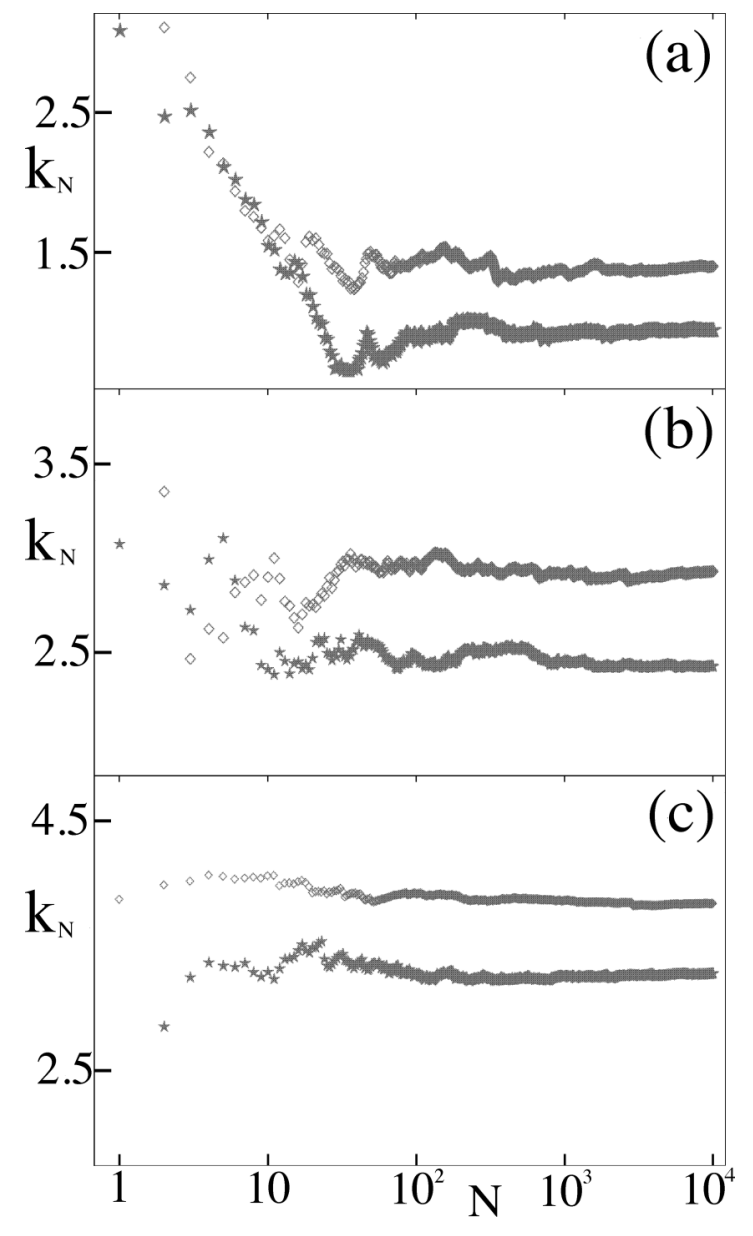

FIG. 5. The Lyapunov exponents for trajectories on the InM (stars) and in the 3D unit cell (diamonds) for coupling constants and energies: (a) $a=0.1$ and $E=51$ d.u.; (b) $a=0.5$ and $E=64$ d.u.; (c) $a=1.0$ and $E=84$ d.u. All energies are above the high saddle so trajectories are free to move throughout the optical lattice, regardless of whether they are on the InM or off of it.

$\mathbf{Y}_{2,0}=\mathbf{X}_{2,0}+\frac{d_{0}}{d_{2}} \mathbf{d}_{2}$, where $\mathbf{X}_{2,0}=\mathbf{X}_{1, \tau}$, and evolve this new set of points for one time step $\tau$. This process is repeated $N$ times, generating a sequence of distances $d_{j}=\left|\mathbf{d}_{j}\right|$, for $j=1, \ldots N$. The Lyapunov exponent is then defined as

$$
k_{N}=\frac{1}{N \tau} \sum_{j=1}^{N} \ln \left(\frac{\mathbf{d}_{j}}{\mathbf{d}_{0}}\right) .
$$

If $\tau$ is chosen small enough, then the Lyapunov exponent $k_{N}$ will be independent of $\tau$ and $d_{0}$. If $\mathbf{X}_{0,0}$ is in a regular region of the phase space, then $\lim _{N \rightarrow \infty} k_{N}=0$. If $\mathbf{X}_{0,0}$ is in a chaotic region of the phase space, then $\lim _{N \rightarrow \infty} k_{N}$ approaches a positive value [19,21].

In Fig. 5, we show the values of the dominant Lyapunov exponent $k_{N}$ for $1 \leqslant N \leqslant 10^{5}, \tau=2$ d.u., and $d_{0}=5 \times 10^{-3}$ d.u., for initial conditions taken both on the InM and off the InM. The energies used to compute the Lyapunov exponents are the same as those in Figs. 4(c), 4(f), and 4(i). The initial conditions, for trajectories on and off the InM, are the same except for trajectories on the InM initially $x=0, p_{x}=0$ and for trajectories off the InM initially $x=10^{-5}$ d.u., $p_{x}=10^{-6}$ d.u.

In Fig. 5(a), $a=0.1, E=51$ d.u. and the "star" represents the initial condition is given by $\left(x=0, y=z=1.6618, p_{x}=\right.$ $\left.0, p_{y}=2.9, p_{z}=4.791\right)$ in d.u. The "diamond" represents the initial condition is given by $\left(x=10^{-5}, y=z=1.6618, p_{x}=\right.$ $\left.10^{-6}, p_{y}=2.9, p_{z}=4.791\right)$ in d.u.

In Fig. 5(b), $a=0.5, E=64$ d.u. and the "star" represents the initial condition is given by $\left(x=0, y=z=1.9106, p_{x}=\right.$ $\left.0, p_{y}=5.515, p_{z}=4.5\right)$ in d.u. The "diamond" represents the initial condition is given by $\left(x=10^{-5}, y=z=1.9106, p_{x}=\right.$ $\left.10^{-6}, p_{y}=5.515, p_{z}=4.5\right)$ in d.u.

In Fig. 5(c), $a=1, E=84$ d.u. and the "star" represents the initial condition is given by $\left(x=0, y=z=2.0945, p_{x}=\right.$ $\left.0, p_{y}=8.5745, p_{z}=-3.2357\right)$ in d.u. The "diamond" represents the initial condition is given by $\left(x=10^{-5}, y=z=\right.$ 2.0945, $\left.p_{x}=10^{-6}, p_{y}=8.5745, p_{z}=-3.2357\right)$ in d.u. In all cases, the Lyapunov exponents approach positive values, indicating that the motion is chaotic. This is confirmed for the trajectories on the InM by the PSSs in Figs. 4(c), 4(f), and 4(i). For all three values of the coupling constant the Lyapunov exponent for the 3D chaotic motion is larger than for the corresponding 2D motion. Also the Lyapunov exponent increases in value as the coupling constant $a$ increases.

\section{QUANTUM DYNAMICS ON THE THREE-DIMENSIONAL LATTICE}

The Schrodinger equation, for noninteracting quantum particles on the optical lattice is

$$
\begin{aligned}
& -\frac{\partial^{2} \psi(x, y, z)}{\partial x^{2}}-\frac{\partial^{2} \psi(x, y, z)}{\partial y^{2}}-\frac{\partial^{2} \psi(x, y, z)}{\partial z^{2}} \\
& +u\left[\cos ^{2}(x)+\cos ^{2}(y)+\cos ^{2}(z)\right] \psi(x, y, z) \\
& +2 a u \cos (x) \cos (y) \psi(x, y, z)+2 a u \cos (x) \cos (z) \psi(x, y, z) \\
& +2 a u \cos (y) \cos (z) \psi(x, y, z)=E \psi(x, y, z)
\end{aligned}
$$

If we note the identity $\cos ^{2}(2 x)=\frac{1}{2}[1+\cos (2 x)]$, we can write the Schrodinger equation in the form

$$
\begin{aligned}
\frac{\partial^{2} \psi(x, y, z)}{\partial x^{2}} & +\frac{\partial^{2} \psi(x, y, z)}{\partial y^{2}}+\frac{\partial^{2} \psi(x, y, z)}{\partial z^{2}}+\epsilon \psi(x, y, z) \\
& -2 q[\cos (2 x)+\cos (2 y)+\cos (2 z)] \psi(x, y, z) \\
& -8 a q \cos (x) \cos (y) \psi(x, y, z) \\
& -8 a q \cos (x) \cos (z) \psi(x, y, z) \\
& -8 a q \cos (y) \cos (z) \psi(x, y, z)=0
\end{aligned}
$$

with $\epsilon=E-\frac{3 u}{2}$ and $q=\frac{u}{4}$. For the case $a=0$ this reduces to

$$
\begin{aligned}
\frac{\partial^{2} \psi(x, y, z)}{\partial x^{2}} & +\frac{\partial^{2} \psi(x, y, z)}{\partial y^{2}}+\frac{\partial^{2} \psi(x, y, z)}{\partial z^{2}}+\epsilon \psi(x, y, z) \\
& -2 q[\cos (2 x)+\cos (2 y)+\cos (2 z)] \\
& \times \psi(x, y, z)=0,
\end{aligned}
$$

so the dynamics in the $x, y$, and $z$ directions decouples and, for each direction, is governed by a Mathieu equation. 
The Floquet-Bloch theorem states that Eq. (12) has solutions of the form

$$
\psi_{a}(x, y, z)=\mathrm{e}^{i\left(k_{x} x+k_{y} y+k_{z} z\right)} U_{a}(x, y, z),
$$

where $k_{x}, k_{y}$, and $k_{z}$ are components of the Bloch momentum, $U_{a}(x, y, z)$ is a periodic function with period $2 \pi$ in the $x$, $y$, and $z$ directions, so that $U_{a}(x+2 \pi, y+2 \pi, z+2 \pi)=$ $U_{a}(x, y, z)$.

For the case $a=0$, the lattice has periodicity $\pi$ in the $x, y$, and $z$ directions. The Floquet-Bloch (F-B) solutions to Eq. (13) (the case $a=0$ ) can be written in the form

$$
\begin{aligned}
\psi_{0}(x, y, z)= & \sum_{n_{x}=-\infty}^{\infty} \sum_{n_{y}=-\infty}^{\infty} \sum_{n_{z}=-\infty}^{\infty} c_{n_{x}} c_{n_{y}} c_{n_{z}} \\
& \times \mathrm{e}^{i\left(k_{x}+2 n_{x}\right) x} \mathrm{e}^{i\left(k_{y}+2 n_{y}\right) y} \mathrm{e}^{i\left(k_{z}+2 n_{z}\right) z} .
\end{aligned}
$$

The F-B solution can also be written as the product of even and odd Mathieu functions, which, for the $x$ direction for example, take the form

$$
\begin{aligned}
C_{0}\left[a_{r}, q, x\right] & \equiv \sum_{n_{x}=-\infty}^{\infty} A_{2 n}^{k_{x}} \cos \left[\left(k_{x}+2 n_{x}\right) x\right] \quad \text { and } \\
S_{0}\left[b_{r}, q, x\right] & \equiv \sum_{n_{x}=-\infty}^{\infty} B_{2 n}^{k_{x}} \sin \left[\left(k_{x}+2 n_{x}\right) x\right],
\end{aligned}
$$

with $\epsilon=a_{r}$ and $\epsilon=b_{r}$, respectively. For a given choice of $q$, the eigenvalues $a_{r}$ and $b_{r}$ take on a countably infinite number of values, and the corresponding Mathieu function is the eigenstate of the system for that eigenvalue.

For the case $a \neq 0$, the lattice has periodicity $2 \pi$ in the $x, y$, and $z$ directions and we can use the Mathieu functions

$$
\begin{aligned}
C_{a}\left[a_{r}, q, x\right] & \equiv \sum_{n_{x}=-\infty}^{\infty} A_{n}^{k_{x}} \cos \left[\left(k_{x}+n_{x}\right) x\right] \quad \text { and } \\
S_{a}\left[b_{r}, q, x\right] & \equiv \sum_{n_{x}=-\infty}^{\infty} B_{n}^{k_{x}} \sin \left[\left(k_{x}+n_{x}\right) x\right]
\end{aligned}
$$

(and similar functions for the $y$ and $z$ directions) to build the Hamiltonian matrix for the lattice. We then diagonalize the Hamiltonian matrix to obtain the energy eigenvalues and eigenfunctions. For the subsequent discussion we only consider eigenvalues and eigenfunctions of the interacting system obtained by using the Mathieu functions $C_{a}\left[a_{r}, q, x\right]$, $C_{a}\left[a_{r}, q, y\right]$, and $C_{a}\left[a_{r}, q, z\right]$ as the basis set. Using only odd Mathieu functions or combinations of even and odd Mathieu functions will give additional bands but not qualitatively different behavior of the bands.

The Brillioun zone for a body-centered cubic crystal is shown in Fig. 6(a), and the symmetry points $\Gamma, H, N$, and $P$ are shown. We will consider a lattice containing 20 unit cells along each spatial direction, and we use period boundary conditions so the Bloch vector takes values $\mathbf{k}=\frac{n_{x}}{20} \hat{x}+\frac{n_{y}}{20} \hat{y}+$ $\frac{n_{z}}{20} \hat{z}$, where $n_{x}, n_{y}$, and $n_{z}$ are integers that can each take values $\{0,1,2, \ldots 20\}$ and $\hat{x}, \hat{y}$, and $\hat{z}$ are unit vectors. It will be useful to compare our results for the band structure of the optical lattice to that of an "empty" BCC lattice, which is obtained by diagonalizing the Hamiltonian matrix using Mathieu functions as the basis set and setting $a=0$ and $u=0$. In Figs. 6(b)

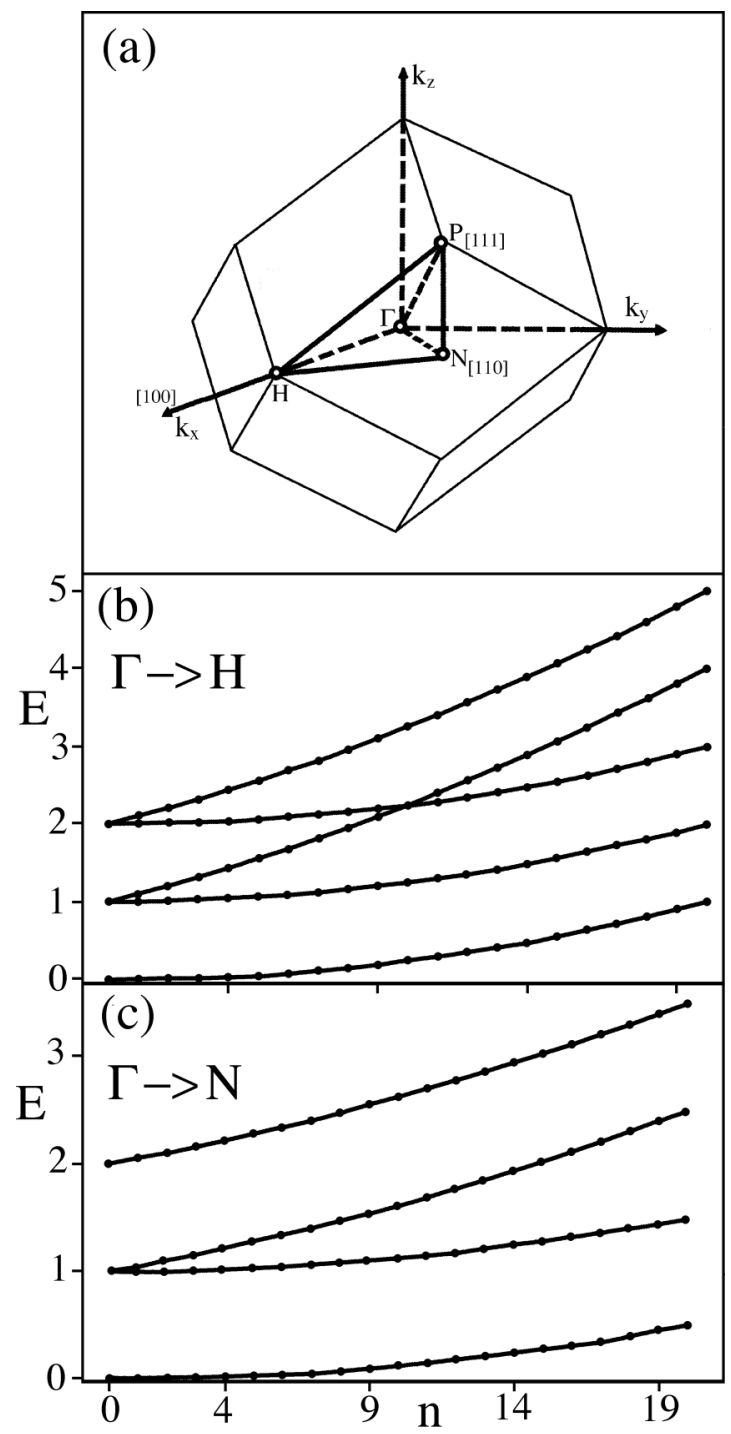

FIG. 6. (a) The Brillioun zone; (b) The $\Gamma-H$ lowest six "empty lattice" energy bands; (c) the $\Gamma-N$ lowest six "empty lattice" energy bands, for a body centered cubic lattice (all in d.u.).

and $6(\mathrm{c})$, we show the lowest six bands for Bloch momentum taken along the $\Gamma-H$ and $\Gamma-N$ directions, respectively. Along the $\Gamma-H$ direction, the Bloch momentum is $\mathbf{k}_{\Gamma H}=\frac{n}{20} \hat{x}$ and along the $\Gamma-N$ direction it is $\mathbf{k}_{\Gamma N}=\frac{n}{40} \hat{x}+\frac{n}{40} \hat{y}$, where $n=0,1, \ldots, 20$ for both cases. We obtain the usual empty lattice band structure for the BCC lattice [5].

In Figs. 7(a)-7(c), we show the six bands with lowest energy for the optical lattice along the $\Gamma-H$ direction of the Brillioun zone for $u=20$ d.u. and coupling constants $a=0.1, a=0.5$, and $a=1.0$, respectively. In Figs. 8(a)-8(c), we show the lowest six bands of the optical lattice along the $\Gamma-N$ direction of the Brillioun zone for $u=20$ and coupling constants $a=$ $0.1, a=0.5$, and $a=1.0$. We find that, for $a<0.5$, the lowest bands, which would be the ones occupied on a BCC optical lattice at low temperature, are flat, indicating that the particles cannot move through the lattice and do not see the crystal structure. The case $a=1.0$ is very different. The particles are chaotic and are free to move through the lattice. They also 

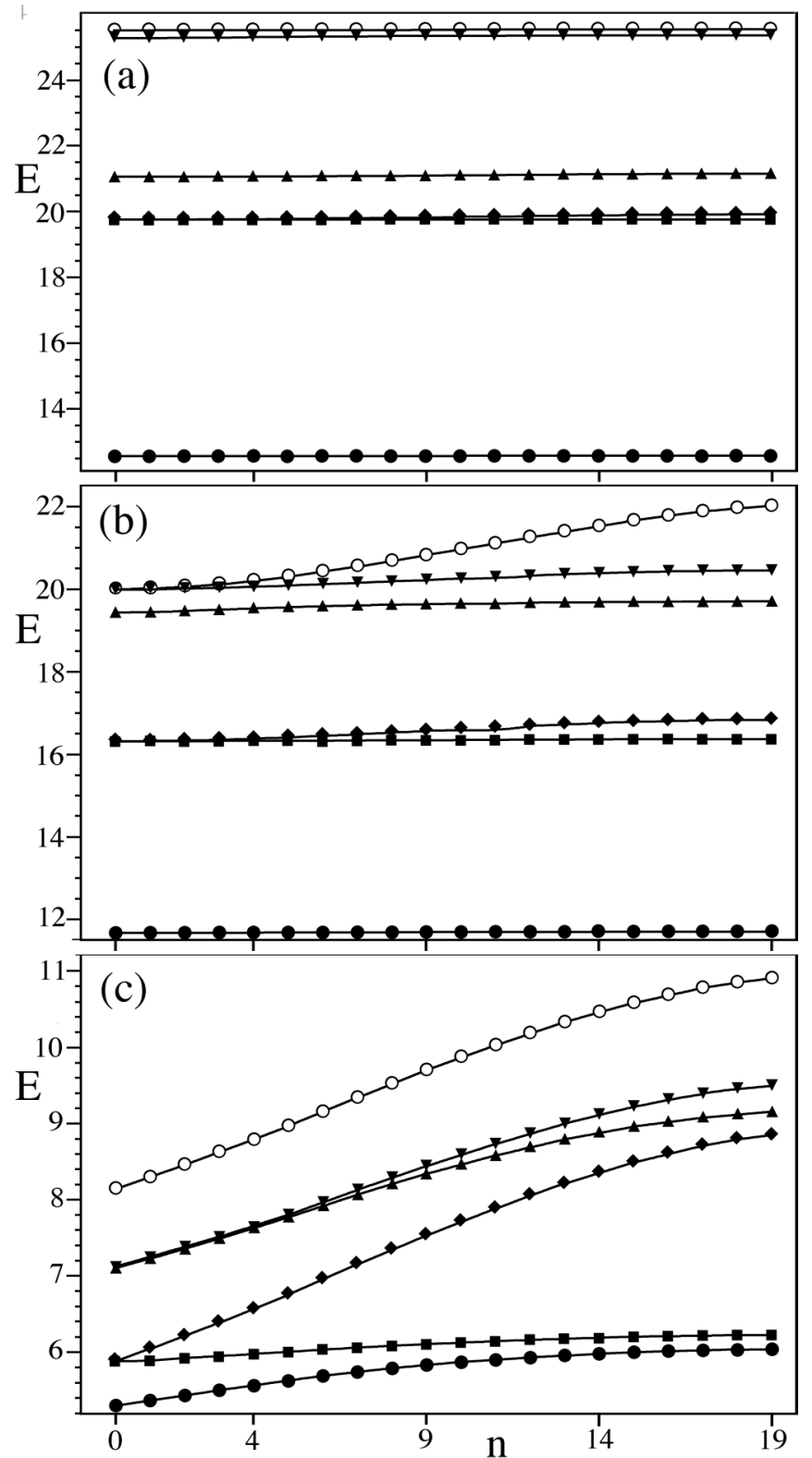

FIG. 7. (a) The lowest six bands, for the $\Gamma-H$ direction of the Brillioun zone, for an optical lattice with periodicity $40 \pi$ in each direction: (a) $a=0.1$; (b) $a=0.5$; (c) $a=1.0$ (all in d.u.).

have band structure more consistent with the band structure of the "empty" lattice, but with fewer degeneracies.

The Fig. 9, we show the Lyapunov exponents for the $3 \mathrm{D}$ optical lattice for energies corresponding to the lowest bands in Figs. 7 and 8. In Fig. 9(a) we show the Lyapunov exponents for $a=0.1$ and $E=13$, with initial condition given by $\left(x=1.5, y=1.66183, z=1.67, p_{x}=\right.$ 2.8, $\left.p_{z}=0.70914, p_{y}=2.0526\right)$ in d.u. In Figs. 7(a) and 8(a), the lowest bands lie at this energy and are completely flat. The Lyapunov exponents indicate that there is no chaos classically in this energy region. In Fig. 9(b), we show the Lyapunov exponents for the case $a=0.5, E=12$ d.u., with the initial condition $\left(x=1.9, y=3 \pi / 2, z=1.8, p_{x}=\right.$ 1.2, $\left.p_{z}=2.0, p_{y}=1.4029\right)$ in d.u. In Figs. 7(b) and 8(b), the lowest bands lie at this energy and again are completely flat,
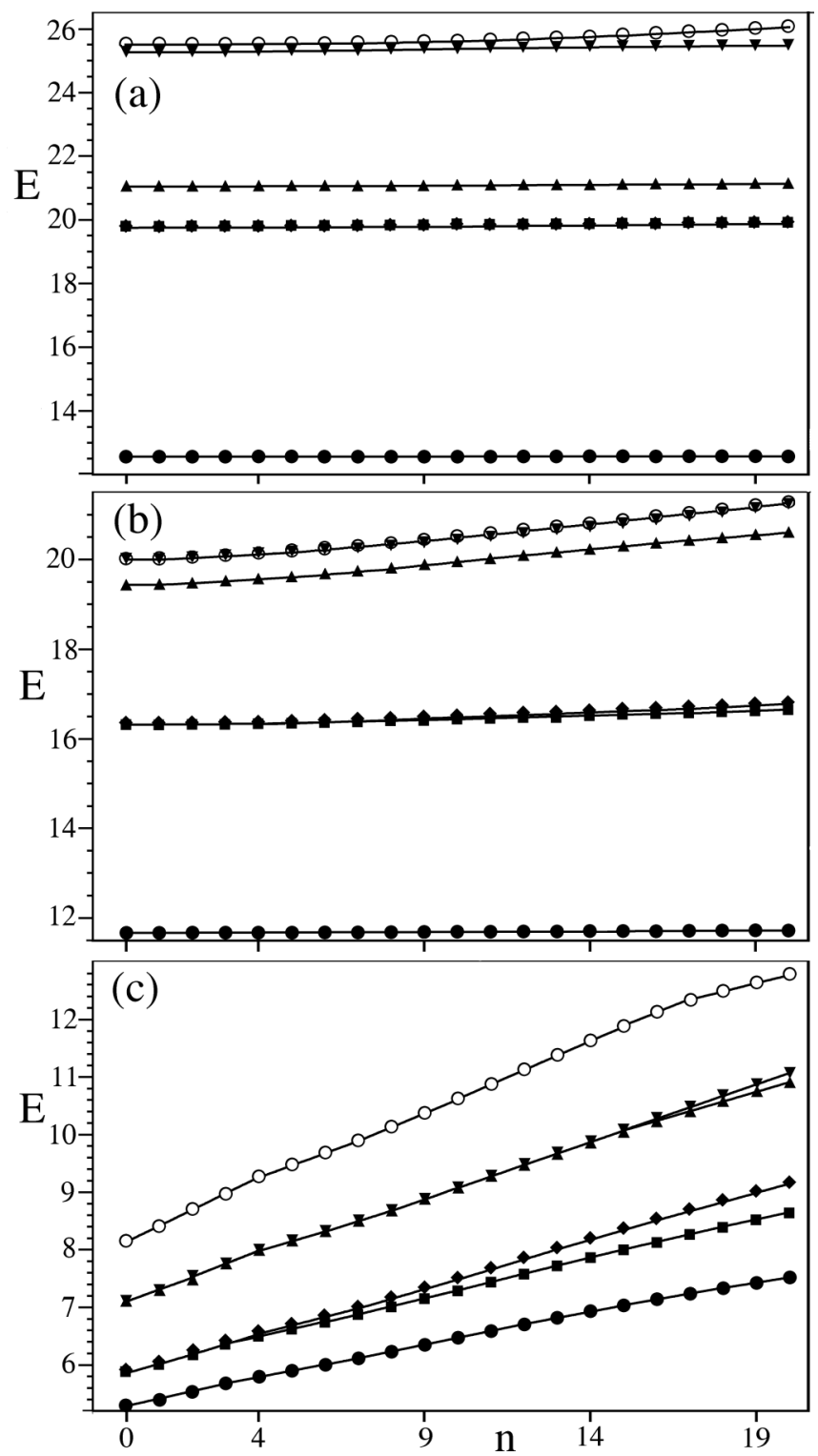

FIG. 8. (a) The lowest six bands, for the $\Gamma-N$ direction of the Brillioun zone, for an optical lattice with periodicity $40 \pi$ in each direction: (a) $a=0.1$; (b) $a=0.5$; (c) $a=1.0$ (all in d.u.).

while the Lyapunov exponent indicates, again, that there is no chaos in this region of the phase space. In both cases, the lowest energy levels are widely spaced in energy and there are large direct band gaps. At the lowest energies, the bands are flat.

Now let us consider the case $a=1$. In Fig. 9(c) we show the Lyapunov exponents for energy $E=5$ d.u., which is the energy of the lowest band in Figs. 7(c) and 8(c). The initial condition is given by $x=1.6, y=1.65, z=$ $\left.3 \pi / 2, p_{x}=0.8, p_{z}=1.2, p_{y}=2.048\right)$ in d.u. Lyapunov exponents approach a positive value, indicating that the phase space dynamics is chaotic in this energy region. The energy levels are much more closely spaced and there are no indirect band gaps. The bands are not flat and the particles can move. The bands look similar to the empty lattice bands, except that many of the empty lattice degeneracies are removed. For this case, the particles again can move through the lattice and the band structure reflects that fact. 


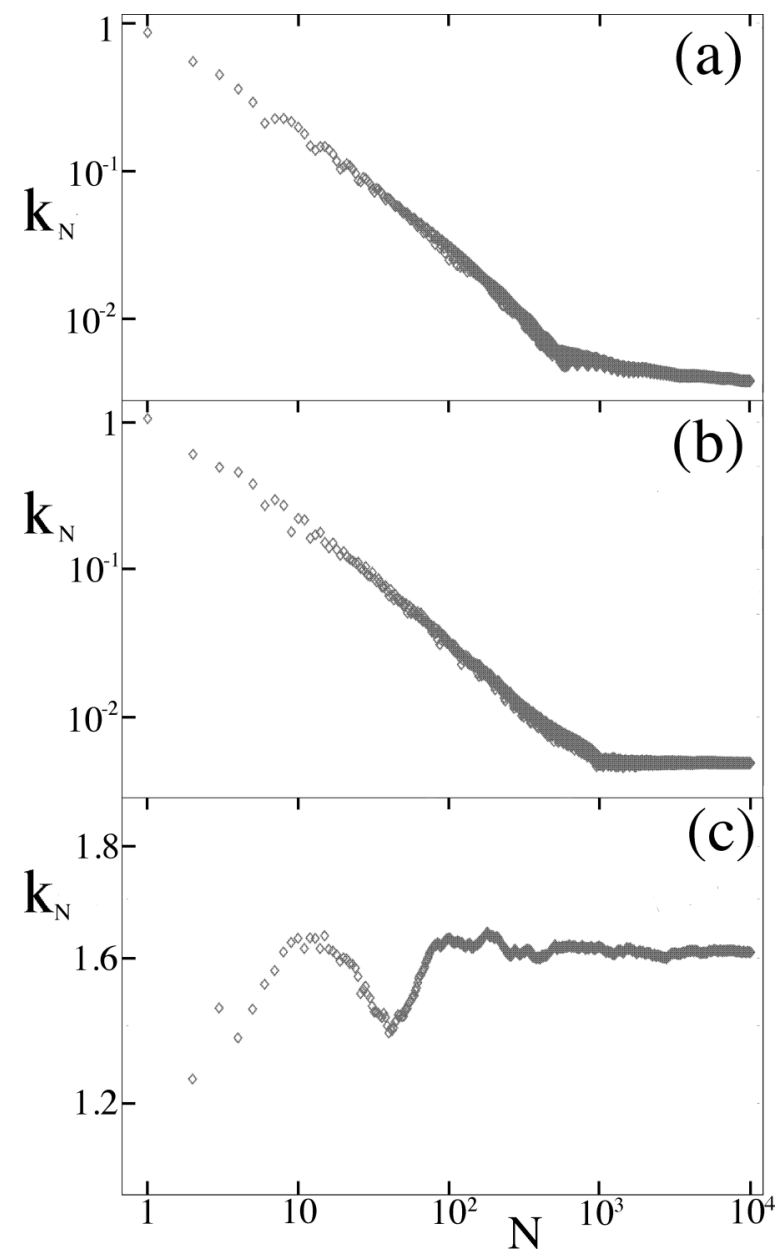

FIG. 9. The Lyapunov exponents for trajectories in the 3D unit cell (diamonds) for coupling constants and energies: (a) $a=0.1$ and $E=13$ d.u.; (b) $a=0.5$ and $E=12$ d.u.; (c) $a=1.0$ and $E=5$ d.u. All energies are above the high saddle so trajectories are free to move throughout the optical lattice, regardless of whether they are on the InM or off of it.

\section{CONCLUSIONS}

We have found evidence, for the case of particle matter waves propagating through a BCC optical lattice, that the band structure associated with this wave propagation is sensitive to the level of classical chaos experienced by the particles. This could have implications for the ability of particles at low energy to thermalize, since thermal motion requires ergodicity in the underlying dynamics.

The lattice we consider here is a body-centered-cubic lattice because it has discrete translational invariance characteristic of such lattices. However, the detailed form of the potential, inside the unit cell, determines the nature of the particle dynamics locally. For an integrable lattice, there are additional constants of the motion that constrain the dynamics. As coupling is turned on, nonlinear resonances between degrees of freedom of the particles can destroy these local constants of the motion. The manner in which this happens depends on the detailed structure of the potential energy inside the unit cell and will be different for every system with local differences in the potential energy.
The results we obtain here seem to indicate that the destruction of local constants of the motion, as signaled by the transition to chaos, allows the particles to fully explore the global symmetries of the lattice. It would be interesting to see if the existence of local constants of the motion inside the unit cell of real solids could explain as yet unexplained anomalies in band structure. It would also be interesting to see if similar effects can be seen in the band structure of electromagnetic and acoustic waves in periodic lattices.

\section{ACKNOWLEDGMENT}

The authors thank the Welch Foundation (Grant No. F1051) for support of this work, and we thank Christof Jung for very helpful discussions.

\section{APPENDIX}

The electric field, due to the three pairs of counterpropagating laser beams, can be written

$$
\begin{aligned}
\mathbf{E}(x, y, z, t)= & {\left[\mathbf{A}^{(x)}(x, t)+\mathbf{A}^{(y)}(y, t)+\mathbf{A}^{(z)}(z, t)\right] e^{-i \omega_{L} t} } \\
& +\left[\mathbf{A}^{(x)}(x, t)+\mathbf{A}^{(y)}(y, t)+\mathbf{A}^{(z)}(z, t)\right]^{*} e^{i \omega_{L} t}
\end{aligned}
$$

The particular form of $\mathbf{A}(\mathbf{r}, t)$ will depend on the lasers. Let us assume that the total electric field amplitude $\mathbf{E}(x, y, t)$ is superposition of the electric fields due to two counterpropagating lasers along the $x$ direction, two counterpropagating lasers along the $y$ direction, and two counterpropagating lasers along the $z$ direction. The lasers propagating along the $x, y$, and $z$ directions have polarizations $\hat{\epsilon}^{(x)}=\cos \left(\theta_{x}\right) \hat{z}+\sin \left(\theta_{x}\right) \hat{y}$, $\hat{\epsilon}^{(y)}=\cos \left(\theta_{y}\right) \hat{z}+\sin \left(\theta_{y}\right) \hat{x}$, and $\hat{\epsilon}^{(z)}=\cos \left(\theta_{z}\right) \hat{x}+\sin \left(\theta_{z}\right) \hat{y}$, respectively. Assume that the lasers form standing waves along their respective directions. The standing wave along the $x$ direction is

$$
\begin{aligned}
\mathbf{A}^{(x)}(x, t)= & \frac{E}{2} \hat{\epsilon}^{(x)} \exp \left[+i\left(k_{L} x+\phi_{x}\right)\right] \\
& +\frac{E}{2} \hat{\epsilon}^{(x)} \exp \left[-i\left(k_{L} x+\phi_{x}\right)\right],
\end{aligned}
$$

with similar expressions for $\mathbf{A}^{(y)}(y, t)$ and $\mathbf{A}^{(z)}(z, t)$. We then can write

$$
\begin{aligned}
\mid \mathbf{A}^{(x)} & (x, t)+\mathbf{A}^{(y)}(y, t)++\left.\mathbf{A}^{(z)}(z, t)\right|^{2} \\
= & E^{2}\left[\cos ^{2}\left(k_{x} x\right)+\cos ^{2}\left(k_{y} y\right)+\cos ^{2}\left(k_{z} z\right)\right. \\
& +2 \hat{\epsilon}^{(x)} \hat{\epsilon}^{(y)} \cos \left(k_{x} x\right) \cos \left(k_{y} y\right) \\
& +2 \hat{\epsilon}^{(x)} \hat{\epsilon}^{(z)} \cos \left(k_{x} x\right) \cos \left(k_{z} z\right) \\
& \left.+2 \hat{\epsilon}^{(y)} \hat{\epsilon}^{(z)} \cos \left(k_{y} y\right) \cos \left(k_{z} z\right)\right]
\end{aligned}
$$

where we have assumed the lasers are in phase and we set $\phi_{x}=\phi_{y}=\phi_{z}=0$.

The effective Hamiltonian that governs the dynamics of the alkali atoms in the optical lattice can be written [17]

$$
H_{\mathrm{eff}}=\frac{p^{2}}{2 m}-\frac{|\mathbf{d} \cdot \mathbf{A}(\mathbf{r}, t)|^{2}}{\hbar \Delta},
$$


when the lasers are detuned from resonance with the atoms. The effective Hamiltonian picks up the component of d along the direction of $\mathbf{A}$. Thus, we write $\mathbf{d} \cdot \mathbf{A}=d_{A} A$ and the
Hamiltonian takes the from

$$
H_{\mathrm{eff}}=\frac{p^{2}}{2 m}-d_{A}^{2} \frac{|\mathbf{A}(\mathbf{r}, t)|^{2}}{\hbar \Delta} .
$$

[1] L. E. Reichl, The Transition to Chaos, 2nd ed. (Springer-Verlag, New York, 2004).

[2] S. Sridhar, Phys. Rev. Lett. 67, 785 (1991).

[3] J. Stein and H.-J. Stöckmann, Phys. Rev. Lett. 68, 2867 (1992).

[4] A. L. Virovlyansky, D. V. Makarov, and S. V. Prants, Phys. Uspekhi 55, 18 (2012).

[5] N. W. Ashcroft and N. D. Mermin, Solid State Physics (Holt, Rinehart and Winston, Philadelphia, 1976).

[6] K. M. Ho, C. T. Chan, and C. M. Soukoulis, Phys. Rev. Lett. 65, 3152 (1990).

[7] S. G. Johnson and J. D. Joannopoulos, App. Phys. Lett. 77, 3490 (2000).

[8] D. Garcia-Pablos, M. Sigalas, F. R. Montero de Espinosa, M. Torres, M. Kafesaki, and N. Garcia, Phys. Rev. Lett. 84, 4349 (2000).

[9] A. Hemmerich, D. Schropp, and T. W. Hansch, Phys. Rev. A 44, 1910 (1991).

[10] M. Greiner, I. Bloch, O. Mandel, T. W. Hansch, and T. Esslinger, Appl. Phys. B 73, 769 (2001).
[11] I. Bloch, J. Dalibard, and S. Nascimbene, Nature Phys. 8, 267 (2012).

[12] M. Greiner, O. Mandel, T. Esslinger, T. W. Hansch, and I. Bloch, Nature 415, 39 (2002).

[13] M. Greiner, I Bloch, O. Mandel, T. W. Hänsch, and T. Esslinger, Phys. Rev. Lett. 87, 160405 (2001).

[14] E. Horsley, S. Koppell, and L. E. Reichl, Phys. Rev. E 89, 012917 (2014).

[15] I. Bloch, Nature Phys. 1, 25 (2005).

[16] M. Lewenstein, A. Sanpera, V. Ahufinger, B. Damski, A. Sen, and U. Sen, Adv. Phys. 56, 243 (2007).

[17] R. Graham, M. Schlautmann, and P. Zoller, Phys. Rev. A 45, R19(R) (1992).

[18] S. Wiggins, Normally Hyperbolic Invariant Manifolds in Dynamical Systems (Springer Verlag, Berlin, 1994).

[19] G. Benettin, L. Galgani, and J. M. Strelcyn, Phys. Rev. A 14, 2338 (1976).

[20] G. Benettin, C. Froeschle, and J. P. Scheidecker, Phys. Rev. A 19, 2454 (1979).

[21] M. Casartelli, E. Diana, L. Galgani, and A. Scotti, Phys. Rev. A 13, 1921 (1976). 